\title{
ATTRACTIVITY OF NONLINEAR IMPULSIVE DELAY DIFFERENTIAL EQUATIONS
}

\author{
ZHICHUN YANG AND DAOYI XU
}

Received 12 October 2005; Revised 16 January 2006; Accepted 17 January 2006

The attractivity of nonlinear differential equations with time delays and impulsive effects is discussed. We obtain some criteria to determine the attracting set and attracting basin of the impulsive delay system by developing an impulsive delay differential inequality and introducing the concept of nonlinear measure. Examples and their simulations illustrate the effectiveness of the results and different asymptotical behaviors between the impulsive system and the corresponding continuous system.

Copyright (c) 2006 Z. Yang and D. Xu. This is an open access article distributed under the Creative Commons Attribution License, which permits unrestricted use, distribution, and reproduction in any medium, provided the original work is properly cited.

\section{Introduction}

The stability and attractivity of impulsive differential equations have been deeply investigated in the monographs of Baunnov and Simeonov [1], Lakshmikantham et al. [6], Samoulenko and Perestyuk [16], Borisenko et al. [3]. The recent work has provided a full discussion of this subject for impulsive delay differential equations (see, e.g., Yan and Shen [19], Liu and Ballinger [10], Liu et al. [12], Yu [20], Zhang and Sun [21], etc.). Most of these results on asymptotic behavior are valid locally in the neighborhood of the equilibrium state, but do not estimate the range of the stable region and domain of attraction (referring to the definition given by Lakshmikantham and Leela [7], Šiljak [17], Kolmanovskii and Nosov [5]). That is, we do not know how far initial conditions can be allowed to vary without disrupting the asymptotic properties of the equilibrium state. Furthermore, it may be difficult to know whether the equilibrium state exists in nonlinear impulsive delay systems. In this case, it should be important and interesting to estimate the region attracting solutions of the impulsive systems. Therefore, a general problem of the attractivity is to discuss the attracting set and attracting basin for the impulsive systems. Some significant progress has been made in the techniques and methods of determining the attracting set and attracting basin (domain of attraction) for the continuous systems described by ordinary differential equations $[7,17]$ and functional differential

Hindawi Publishing Corporation

Journal of Applied Mathematics and Stochastic Analysis

Volume 2006, Article ID 83152, Pages 1-12

DOI 10.1155/JAMSA/2006/83152 
equations $[5,9,13,15,18]$. However, so far the corresponding problems for impulsive delay differential equations have not been considered.

In this paper, by developing an impulsive delay differential inequality and introducing the concept of nonlinear measure, we study the attractivity for a class of nonlinear impulsive delay differential equations. The criteria present a feasible and effective approach to estimate the attracting set, attracting basin, and asymptotically stable region of the impulsive systems by solving an algebraic equation. Examples and their simulations are given to demonstrate the effectiveness of our results.

\section{Preliminaries}

Let $\mathbb{N}$ be the set of all positive integers, let $\mathbb{R}^{n}$ be the real $n$-dimensional vector space with a norm $\|\cdot\|$, and let $\mathbb{R}^{m \times n}$ be the set of $m \times n$ real matrices. $\mathbb{R}^{+}=[0,+\infty)$ and $I$ denotes the $n \times n$ unit matrix.

Let $\tau>0$ be the upper bound of time delays and let $t_{1}<\cdots<t_{k}<t_{k+1}<\cdots(k \in \mathbb{N})$ be the fixed points with $\lim _{k \rightarrow \infty} t_{k}=\infty$ (called impulsive moments).

For a function $x=\left(x_{1}, \ldots, x_{n}\right)^{T}: \mathbb{R} \rightarrow \mathbb{R}^{n}$, we define

$$
\begin{gathered}
D^{+} x(t)=\limsup _{s \rightarrow 0^{+}} \frac{x(t+s)-x(t)}{s}, \quad x\left(t^{-}\right)=\lim _{s \rightarrow 0^{-}} x(t+s), \quad x\left(t^{+}\right)=\lim _{s \rightarrow 0^{+}} x(t+s), \\
{[x(t)]_{\tau}=\left(\left[x_{1}(t)\right]_{\tau}, \ldots,\left[x_{n}(t)\right]_{\tau}\right)^{T}, \quad\left[x_{i}(t)\right]_{\tau}=\sup _{-\tau \leq s \leq 0}\left\{x_{i}(t+s)\right\} .}
\end{gathered}
$$

$C[X, Y]$ denotes the space of continuous mappings from the topological space $X$ to the topological space $Y$. Especially, let $C \triangleq C\left[[-\tau, 0], \mathbb{R}^{n}\right]$.

$\mathrm{PC}\left[J, \mathbb{R}^{n}\right] \triangleq\left\{\psi: J \rightarrow \mathbb{R}^{n} \mid \psi(t)\right.$ is continuous at $t \neq t_{k}, \psi\left(t_{k}^{+}\right)$and $\psi\left(t_{k}^{-}\right)$exist, $\psi\left(t_{k}\right)=$ $\psi\left(t_{k}^{+}\right)$, for $\left.t_{k} \in J\right\}$, where $J \subset \mathbb{R}$ is an interval.

$\mathrm{PC} \triangleq\left\{\phi:[-\tau, 0] \rightarrow \mathbb{R}^{n} \mid \phi\left(t^{+}\right)=\phi(t)\right.$ for $t \in[-\tau, 0), \phi\left(t^{-}\right)$exists for $t \in(-\tau, 0]$, $\phi\left(t^{-}\right)=\phi(t)$ for all but at most a finite number of points $\left.t \in(-\tau, 0]\right\}$. PC is a space of piecewise right-hand continuous functions with the norm $\|\phi\|_{\tau}=\sup _{-\tau \leq s \leq 0}\{\|\phi(s)\|\}$, for $\phi \in$ PC.

In this paper, we will consider the following nonlinear impulsive delay differential equations:

$$
\begin{gathered}
\dot{x}(t)=f(x(t))+g\left(t, x_{t}\right), \quad t \neq t_{k}, \\
\Delta x\left(t_{k}\right):=x\left(t_{k}\right)-x\left(t_{k}^{-}\right)=I_{k}\left(x\left(t_{k}^{-}\right)\right), \quad k \in \mathbb{N},
\end{gathered}
$$

where $f \in C\left[\mathbb{R}^{n}, \mathbb{R}^{n}\right], g \in C\left[\left[t_{k-1}, t_{k}\right) \times \mathrm{PC}, \mathbb{R}^{n}\right]$ and the limit $\lim _{(t, \phi) \rightarrow\left(t_{k}^{-}, \varphi\right)} g(t, \phi)=$ $g\left(t_{k}^{-}, \varphi\right)$ exists, $I_{k} \in C\left[\mathbb{R}^{n}, \mathbb{R}^{n}\right], x_{t} \in \mathrm{PC}$ is defined by $x_{t}(s)=x(t+s), s \in[-\tau, 0], \dot{x}(t)$ denotes the right-hand derivative of $x(t)$.

Definition 2.1. A function $x:\left[t_{0}-\tau, \infty\right) \rightarrow \mathbb{R}^{n}$ is called a solution of $(2.2)$ through $\left(t_{0}, \phi\right)$ if $x \in \mathrm{PC}\left[\left[t_{0}, \infty\right), \mathbb{R}^{n}\right]$ as $t \geq t_{0}$, and satisfies (2.2) with the initial condition

$$
x\left(t_{0}+s\right)=\phi(s), \quad \phi \in \mathrm{PC}, s \in[-\tau, 0] .
$$


Throughout the paper, we always assume that for any $\phi \in \mathrm{PC}$, system (2.2) has at least one solution through $\left(t_{0}, \phi\right)$, denoted by $x\left(t, t_{0}, \phi\right)$ or $x_{t}\left(t_{0}, \phi\right)$, where $x_{t}\left(t_{0}, \phi\right)(s)=x(t+$ $\left.s, t_{0}, \phi\right), s \in[-\tau, 0]$. Clearly, $x_{t}\left(t_{0}, \phi\right) \in \mathrm{PC}$ for $t \geq t_{0}$. For more details on the existence of solutions of impulsive delay differential equations, one refers to Liu and Ballinger [11], Bănov and Stamova [2].

Definition 2.2. A set $S \subset \mathrm{PC}$ is called an attracting set of (2.2) and $D \subset \mathrm{PC}$ is called an attracting basin of $S$ if for any initial value $\phi \in D$, the solution $x_{t}\left(t_{0}, \phi\right)$ converges to $S$ as $t \rightarrow+\infty$. That is,

$$
\operatorname{dist}\left(x_{t}\left(t_{0}, \phi\right), S\right) \longrightarrow 0, \quad \text { as } t \longrightarrow+\infty \text {, }
$$

where $\operatorname{dist}(\varphi, S)=\inf _{\psi \in S} \operatorname{dist}(\varphi, \psi), \operatorname{dist}(\varphi, \psi)=\sup _{s \in[-\tau, 0]}\|\varphi(s)-\psi(s)\|$, for $\varphi \in$ PC.

Especially, the set $S$ is called a global attracting set of (2.2) if $D=$ PC. The set $D$ is called a domain of attraction if $x=0$ is a solution of (2.2) and the zero solution (i.e., $S=\{0\}$ ) attracts solutions $x\left(t, t_{0}, \phi\right)$ for all $\phi \in D$. Moreover, if the zero solution is stable, we call $D$ an asymptotically stable region of (2.2).

In order to introduce the concept of the nonlinear measure, we recall the matrix norms $\|A\|$ and the matrix measure $\mu(A)$ introduced by the vector norm $\|\cdot\|$ as follows:

$$
\|A\|=\sup _{x \neq 0, x \in \mathbb{R}^{n}} \frac{\|A x\|}{\|x\|}, \quad \mu(A)=\lim _{s \rightarrow 0^{+}} \frac{\|I+s A\|-1}{s}, \quad A \in \mathbb{R}^{n \times n} .
$$

Now, based on (2.5), we define the nonlinear measure as follows.

Definition 2.3. For a function $F: \mathbb{R}^{n} \rightarrow \mathbb{R}^{n}$, call

$$
\mu(F)=\sup _{x \neq 0, x \in \mathbb{R}^{n}} \limsup _{s \rightarrow 0^{+}} \frac{\|x+s F(x)\|-\|x\|}{s\|x\|}
$$

the nonlinear measure of $F$.

Obviously, $-\infty<\mu(F) \leq+\infty$. Especially, if $F(x)=A x, A \in \mathbb{R}^{n \times n}$, then $\mu(F)=\mu(A)$, where $\mu(A)$ is the matrix measure. Therefore, the concept of the nonlinear measure actually is an extension of the matrix measure (see also, Kolmanovskii and Myshkis [4], Qiao et al. [14]). According to the definition, we easily verify the following.

Lemma 2.4. Let $\mu(F), \mu(G)<+\infty$. Then,

(i) $\mu(\lambda F)=\lambda \mu(F)$, where $\lambda \geq 0$;

(ii) $\mu(F+G) \leq \mu(F)+\mu(G)$;

(iii) $-L(F) \leq \mu(F) \leq L(F)$, where the constant $L(F)=\sup _{x \neq 0, x \in \mathbb{R}^{n}}(\|F(x)\| /\|x\|)$.

The following result on the impulsive delay differential inequality is an extension of the continuous case of Lakshmikantham and Leela [8, Theorem 6.9.1], and will play an important role in the qualitative analysis of impulsive delay differential equations in Section 3. 
4 Attractivity of impulsive differential equations

Lemma 2.5. Let $F:\left[t_{0}-\tau, \infty\right) \times \mathbb{R} \times \mathbb{R} \rightarrow \mathbb{R}$ be such that $F(t, x, \cdot)$ is nondecreasing for each $(t, x)$ and let $I_{k}: \mathbb{R} \rightarrow \mathbb{R}$ be nondecreasing. Suppose $u, v \in \mathrm{PC}\left[\left[t_{0}-\tau, \infty\right), \mathbb{R}\right]$ satisfy

$$
\begin{gathered}
D^{+} u(t) \leq F\left(t, u(t),[u(t)]_{\tau}\right), \quad t \geq t_{0}, \\
u\left(t_{k}\right) \leq I_{k}\left(u\left(t_{k}^{-}\right)\right), \quad k \in \mathbb{N}, \\
D^{+} v(t)>F\left(t, v(t),[v(t)]_{\tau}\right), \quad t \geq t_{0}, \\
v\left(t_{k}\right) \geq I_{k}\left(v\left(t_{k}^{-}\right)\right), \quad k \in \mathbb{N} .
\end{gathered}
$$

Then $u(t) \leq v(t)$, for $t_{0}-\tau \leq t \leq t_{0}$ implies that $u(t) \leq v(t)$, for $t \geq t_{0}$.

Proof. We will first prove that

$$
u(t) \leq v(t), \quad t \in\left[t_{0}, t_{1}\right)
$$

If the assertion (2.8) is false, by using the continuity of $u(t), v(t)$ for $t \in\left[t_{0}, t_{1}\right)$ and $u(t) \leq$ $v(t)$ for $t \in\left[t_{0}-\tau, t_{0}\right]$, then there must exist a number $t^{*} \in\left[t_{0}, t_{1}\right)$ such that

$$
\begin{gathered}
u\left(t^{*}\right)=v\left(t^{*}\right), \quad u(t) \leq v(t), \quad t \leq t^{*}, \\
D^{+} u\left(t^{*}\right) \geq D^{+} v\left(t^{*}\right) .
\end{gathered}
$$

In view of (2.9) and the monotonic character of $F$, we have

$$
\begin{aligned}
D^{+} u\left(t^{*}\right) & \leq F\left(t^{*}, u\left(t^{*}\right),\left[u\left(t^{*}\right)\right]_{\tau}\right) \\
& \leq F\left(t^{*}, u\left(t^{*}\right),\left[v\left(t^{*}\right)\right]_{\tau}\right) \\
& =F\left(t^{*}, v\left(t^{*}\right),\left[v\left(t^{*}\right)\right]_{\tau}\right)<D^{+} v\left(t^{*}\right) .
\end{aligned}
$$

This contradicts the inequality (2.10), and so (2.8) holds. Suppose that for $k=1,2, \ldots, m$

$$
u(t) \leq v(t), \quad t \in\left[t_{k-1}, t_{k}\right)
$$

Then

$$
u(t) \leq v(t), \quad t_{m}-\tau \leq t<t_{m}, \quad u\left(t_{m}^{-}\right) \leq v\left(t_{m}^{-}\right)
$$

It is clear from the monotonicity of $I_{m}$ that

$$
u\left(t_{m}\right) \leq I_{m}\left(u\left(t_{m}^{-}\right)\right) \leq I_{m}\left(v\left(t_{m}^{-}\right)\right) \leq v\left(t_{m}\right) .
$$

Thus, $u(t) \leq v(t)$ for $t_{m}-\tau \leq t \leq t_{m}$. Employing the similar process of the proof of (2.8), we have $u(t) \leq v(t)$, for $t \in\left[t_{m}, t_{m+1}\right)$. By the induction, the conclusion holds and the proof is complete. 


\section{Main results}

In this paper, we always suppose the following.

$\left(\mathrm{A}_{1}\right) \mu(f)<+\infty$ and $\|g(t, \varphi)\| \leq p\left(\|\varphi\|_{\tau}\right)$ for any $\varphi \in \mathrm{PC}, t \geq t_{0}$, where $p: \mathbb{R}_{+} \rightarrow \mathbb{R}_{+}$is continuous and monotonically nondecreasing.

$\left(\mathrm{A}_{2}\right)\left\|x+I_{k}(x)\right\| \leq \alpha\|x\|$ for any $k \in \mathbb{N}$ and $x \in \mathbb{R}^{n}$, where the constant $\alpha>0$.

Theorem 3.1. Let $t_{k}-t_{k-1} \leq \varrho$ for $k \in \mathbb{N}$. Assume that $\left(\mathrm{A}_{1}\right)$ and $\left(\mathrm{A}_{2}\right)$ with $0<\alpha<1$ hold. If there exist two nonnegative constants $z_{1}<z_{2}$ such that for any $z \in\left[z_{1}, z_{2}\right]$

$$
\Psi_{1}(z):=\frac{p(z)}{\alpha}+\left[\mu(f)+\frac{\ln \alpha}{\varrho}\right] z<0,
$$

then $S_{1}=\left\{\phi \in \mathrm{PC} \mid\|\phi\|_{\tau} \leq z_{1}\right\}$ is an attracting set of (2.2) and $D_{1}=\left\{\phi \in \mathrm{PC} \mid\|\phi\|_{\tau}<\right.$ $\left.\alpha z_{2}\right\}$ is an attracting basin of $S_{1}$.

Proof. For any $\phi \in D_{1} \subset \mathrm{PC}$, let $x(t)=\left(t, t_{0}, \phi\right)$ be the solution of (2.2) through $\left(t_{0}, \phi\right)$. By $\left(\mathrm{A}_{1}\right)$, we calculate the right upper derivative along the solution (2.2):

$$
\begin{aligned}
D^{+}\|x(t)\|= & \limsup _{s \rightarrow 0^{+}} \frac{\|x(t+s)\|-\|x(t)\|}{s} \\
\leq & \limsup _{s \rightarrow 0^{+}} \frac{\|x(t)+s f(x(t))\|-\|x(t)\|}{s} \\
& +\limsup _{s \rightarrow 0^{+}} \frac{\|x(t+s)\|-\|x(t)+s f(x(t))\|}{s} \\
\leq & \mu(f)\|x(t)\|+\limsup _{s \rightarrow 0^{+}}\left\|\frac{x(t+s)-x(t)}{s}-f(x(t))\right\| \\
\leq & \mu(f)\|x(t)\|+\left\|g\left(t, x_{t}\right)\right\| \\
\leq & \mu(f)\|x(t)\|+p\left([\|x(t)\|]_{\tau}\right), \quad t \geq t_{0} .
\end{aligned}
$$

On the other hand, by $\left(\mathrm{A}_{2}\right)$,

$$
\left\|x\left(t_{k}^{+}\right)\right\|=\left\|x\left(t_{k}^{-}\right)+I_{k}\left(x\left(t_{k}^{-}\right)\right)\right\| \leq \alpha\left\|x\left(t_{k}^{-}\right)\right\|, \quad k \in \mathbb{N} .
$$

From (3.1), the continuity of $p(z)$ and $\mu(f)+\ln \alpha / \varrho<0$, there must exist an $\epsilon>0$ such that

$$
h(z):=z+\frac{p(z)+\epsilon}{\alpha}\left[\mu(f)+\frac{\ln \alpha}{\varrho}\right]^{-1}>0 \quad \forall z \in\left[z_{1}, z_{2}\right] .
$$

Let $v(t)$ be a solution of

$$
\begin{gathered}
\dot{v}(t)=\mu(f) v(t)+p\left([v(t)]_{\tau}\right)+\epsilon, \quad t \neq t_{k}, t \geq t_{0}, \\
v\left(t_{k}\right)=\alpha v\left(t_{k}^{-}\right), \quad k \in \mathbb{N}, \\
v(t)=\left\|\phi\left(t-t_{0}\right)\right\|, \quad t_{0}-\tau \leq t \leq t_{0} .
\end{gathered}
$$




\section{Attractivity of impulsive differential equations}

Taking $F(t, x, y)=\mu(f) x+p(y)$ and $I_{k}(x)=\alpha x$, from the monotonicity of $p(\cdot)$ and $\alpha>0$, then $F(t, x, y)$ is nondecreasing in $y$ for each $(t, x)$ and $I_{k}(x)$ is nondecreasing in $x$. By (3.2), (3.3), (3.5), and Lemma 2.5 with $u(t)=\|x(t)\|$, we have

$$
0 \leq\|x(t)\| \leq v(t), \quad t \geq t_{0}
$$

By the formula for the variation of parameters, we have

$$
v(t)=W\left(t, t_{0}\right) v\left(t_{0}\right)+\int_{t_{0}}^{t} W(t, s)\left[p\left([v(s)]_{\tau}\right)+\epsilon\right] d s, \quad t \geq t_{0},
$$

where $W(t, s), t, s \geq t_{0}$ is the Cauchy matrix of linear impulsive system (refer to [6]):

$$
\begin{array}{ll}
\dot{\omega}(t)=\mu(f) \omega(t), & t \neq t_{k}, \\
\omega\left(t_{k}\right)=\alpha \omega\left(t_{k}^{-}\right), & k \in \mathbb{N} .
\end{array}
$$

It is easily seen that

$$
W(t, s)=e^{\mu(f)(t-s)} \prod_{s<t_{k} \leq t} \alpha, \quad t \geq s \geq t_{0}
$$

Since $0<\alpha<1$ and $\varrho \geq t_{k}-t_{k-1}$, we have the following estimate:

$$
\begin{aligned}
W(t, s) & \leq e^{\mu(f)(t-s)} \alpha^{[(t-s) / \varrho-1]} \\
& =\frac{1}{\alpha} e^{[\mu(f)+\ln \alpha / \varrho](t-s)}, \quad t \geq s \geq t_{0} .
\end{aligned}
$$

Combining with (3.7), we get

$$
v(t) \leq \frac{\|\phi(0)\|}{\alpha} e^{[\mu(f)+\ln \alpha / \varrho]\left(t-t_{0}\right)}+\int_{t_{0}}^{t} \frac{p\left([v(s)]_{\tau}\right)+\epsilon}{\alpha} e^{[\mu(f) \ln \alpha / \varrho](t-s)} d s .
$$

Since $\phi \in D_{1}$, we have

$$
v(t)=\left\|\phi\left(t-t_{0}\right)\right\|<\alpha z_{2} \leq z_{2}, \quad t_{0}-\tau \leq t \leq t_{0} .
$$

In the following, we will prove that

$$
v(t)<z_{2}, \quad t \geq t_{0}
$$

If this is not true, then by the estimate (3.12) and the piecewise continuity of $v(t)$, there exists a $t^{*}>t_{0}$ satisfying

$$
v\left(t^{*}\right) \geq z_{2}, \quad v(t)<z_{2}, \quad \text { for } t<t^{*} .
$$


By (3.11), (3.12), and (3.14), we have

$$
\begin{aligned}
v\left(t^{*}\right) & \leq \frac{\|\phi(0)\|}{\alpha} e^{[\mu(f)+\ln \alpha / \varrho]\left(t^{*}-t_{0}\right)}+\int_{t_{0}}^{t^{*}} \frac{p\left([v(s)]_{\tau}\right)+\epsilon}{\alpha} e^{[\mu(f)+\ln \alpha / \varrho]\left(t^{*}-s\right)} d s \\
& \leq e^{[\mu(f)+\ln \alpha / \varrho]\left(t^{*}-t_{0}\right)} z_{2}+\frac{p\left(z_{2}\right)+\epsilon}{\alpha} \int_{t_{0}}^{t^{*}} e^{[\mu(f)+\ln \alpha / \varrho]\left(t^{*}-s\right)} d s \\
& =e^{[\mu(f)+\ln \alpha / \varrho]\left(t^{*}-t_{0}\right)} z_{2}-\frac{p\left(z_{2}\right)+\epsilon}{\alpha}\left[\mu(f)+\frac{\ln \alpha}{\varrho}\right]^{-1}\left[1-e^{[\mu(f)+\ln \alpha / \varrho]\left(t^{*}-t_{0}\right)}\right] \\
& =e^{[\mu(f)+\ln \alpha / \varrho]\left(t^{*}-t_{0}\right)}\left\{z_{2}+\frac{p\left(z_{2}\right)+\epsilon}{\alpha}\left[\mu(f)+\frac{\ln \alpha}{\varrho}\right]^{-1}\right\}-\frac{p\left(z_{2}\right)+\epsilon}{\alpha}\left[\mu(f)+\frac{\ln \alpha}{\varrho}\right]^{-1} .
\end{aligned}
$$

In term of (3.4), $\mu(f)+\ln \alpha / \varrho<0$ and $t^{*}>t_{0}$, then

$$
v\left(t^{*}\right)<z_{2}+\frac{p\left(z_{2}\right)+\epsilon}{\alpha}\left[\mu(f)+\frac{\ln \alpha}{\varrho}\right]^{-1}-\frac{p\left(z_{2}\right)+\epsilon}{\alpha}\left[\mu(f)+\frac{\ln \alpha}{\varrho}\right]^{-1}=z_{2} .
$$

This contradicts the first inequality in (3.14), and so the estimate (3.13) holds. Thus,

$$
\eta:=\limsup _{t \rightarrow+\infty} v(t) \leq z_{2} \text {. }
$$

Since $\mu(f)+\ln \alpha / \varrho<0$, for any given $\delta>0$, there must be $T>0$ such that

$$
\int_{T}^{+\infty} e^{[\mu(f)+\ln \alpha / \varrho] s} d s<\delta
$$

Furthermore, for the above positive number $\delta$, there is a $T^{\prime}>t_{0}$ such that

$$
v(t) \leq \eta+\delta, \quad \text { for } t>T^{\prime} .
$$

Employing (3.11), (3.13), (3.18), (3.19), and the monotonicity of $p(\cdot)$, when $t>T+T^{\prime}+$ $\tau$, we have

$$
\begin{aligned}
v(t) \leq & \frac{\|\phi(0)\|}{\alpha} e^{[\mu(f)+\ln \alpha / \varrho]\left(t-t_{0}\right)}+\int_{t_{0}}^{t} \frac{p\left([v(s)]_{\tau}\right)+\epsilon}{\alpha} e^{[\mu(f)+\ln \alpha / \varrho](t-s)} d s \\
\leq & e^{[\mu(f)+\ln \alpha / \varrho]\left(t-t_{0}\right)} z_{2}+\int_{t_{0}}^{t-T} \frac{p\left([v(s)]_{\tau}\right)+\epsilon}{\alpha} e^{[\mu(f)+\ln \alpha / \varrho](t-s)} d s \\
& +\int_{t-T}^{t} \frac{p\left([v(s)]_{\tau}\right)+\epsilon}{\alpha} e^{[\mu(f)+\ln \alpha / \varrho](t-s)} d s \\
\leq & e^{[\mu(f)+\ln \alpha / \varrho]\left(t-t_{0}\right)} z_{2}+\frac{p\left(z_{2}\right)+\epsilon}{\alpha} \int_{T}^{+\infty} e^{[\mu(f)+\ln \alpha / \varrho] s} d s \\
& +\frac{p(\eta+\delta)+\epsilon}{\alpha} \int_{t-T}^{t} e^{[\mu(f)+\ln \alpha / \varrho](t-s)} d s \\
\leq & e^{[\mu(f)+\ln \alpha / \varrho]\left(t-t_{0}\right)} z_{2}+\frac{p\left(z_{2}\right)+\epsilon}{\alpha} \delta-\frac{p(\eta+\delta)+\epsilon}{\alpha}\left[\mu(f)+\frac{\ln \alpha}{\varrho}\right]^{-1}\left[1-e^{[\mu(f)+\ln \alpha / \varrho] T}\right] .
\end{aligned}
$$


Then, by $\mu(f)+\ln \alpha / \varrho<0$,

$$
\eta=\limsup _{t \rightarrow+\infty} v(t) \leq \frac{p\left(z_{2}\right)+\epsilon}{\alpha} \delta-\frac{p(\eta+\delta)+\epsilon}{\alpha}\left[\mu(f)+\frac{\ln \alpha}{\varrho}\right]^{-1} .
$$

Letting $\delta \rightarrow 0^{+}$, we have

$$
h(\eta)=\eta+\frac{p(\eta)+\epsilon}{\alpha}\left[\mu(f)+\frac{\ln \alpha}{\varrho}\right]^{-1} \leq 0 .
$$

Combining with $\eta \leq z_{2}$ and $h(z)>0$ for any $z \in\left[z_{1}, z_{2}\right]$, then $\eta<z_{1}$. From (3.6),

$$
\limsup _{t \rightarrow+\infty}\|x(t)\| \leq \limsup _{t \rightarrow+\infty} v(t) \leq z_{1} .
$$

The conclusion holds and the proof is complete.

Remark 3.2. The above conclusion remains valid even when the inequality (3.1) holds for $z \in\left(z_{1}, z_{2}\right)$. In fact, for an enough small $\epsilon>0$, the inequality (3.1) holds when $z \in$ $\left[z_{1}+\epsilon, z_{2}-\epsilon\right]$. According to Theorem 3.1, $S_{1}^{\prime}=\left\{\phi \in \mathrm{PC} \mid\|\phi\|_{\tau} \leq z_{1}+\epsilon\right\}$ is an attracting set of (2.2) and $D_{1}^{\prime}=\left\{\phi \in \mathrm{PC} \mid\|\phi\|_{\tau}<\alpha\left(z_{2}-\epsilon\right)\right\}$ is an attracting basin of $S_{1}^{\prime}$. Letting $\epsilon \rightarrow 0^{+}$, we can obtain the conclusion.

According to Theorem 3.1 and Remark 3.2, we have the following corollaries.

Corollary 3.3. Let $t_{k}-t_{k-1} \leq \varrho$ for $k \in \mathbb{N}$. Suppose that $\left(A_{1}\right)$ and $\left(A_{2}\right)$ with $0<\alpha<1$ and $p(z)=\beta z+\gamma$ hold, where $\beta, \gamma \geq 0$. If $\beta / \alpha+\ln \alpha / \varrho+\mu(f)<0$, then $S_{1}=\left\{\phi \in \mathrm{PC} \mid\|\phi\|_{\tau} \leq\right.$ $\left.z_{1}=-(\gamma / \alpha)[\beta / \alpha+\ln \alpha / \varrho+\mu(f)]^{-1}\right\}$ is a globally attracting set of (2.2).

Proof. It is obvious that

$$
\Psi_{1}(z)=\left[\frac{\beta}{\alpha}+\frac{\ln \alpha}{\varrho}+\mu(f)\right] z+\frac{\gamma}{\alpha}<0, \quad z \in\left(z_{1},+\infty\right) .
$$

According to the above results, $S_{1}=\left\{\phi \in \mathrm{PC} \mid\|\phi\|_{\tau} \leq z_{1}\right\}$ is a globally attracting set of (2.2).

Corollary 3.4. Let $x=0$ be a solution of (2.2). If all the conditions in Theorem 3.1 are satisfied except that the inequality (3.1) holds for $z \in\left(0, z_{2}\right)$, then the zero solution is asymptotically stable and $D_{1}=\left\{\phi \in \mathrm{PC} \mid\|\phi\|_{\tau}<\alpha z_{2}\right\}$ is an asymptotically stable region of (2.2).

Proof. According to Theorem 3.1 and Remark 3.2, the zero solution attracts solutions $x\left(t, t_{0}, \phi\right)$ for all $\phi \in D_{1}$ and $D_{1}$ is a domain of attraction. Furthermore, for any given $z_{\epsilon} \in\left(0, z_{2}\right]$ and $\phi \in D_{\epsilon}=\left\{\phi \in \mathrm{PC} \mid\|\phi\|_{\tau}<\alpha z_{\epsilon}\right\}$, we can refer to the proof of (3.13) and obtain that

$$
\left\|x\left(t, t_{0}, \phi\right)\right\|<z_{\epsilon}, \quad t \geq t_{0} .
$$

This implies that the zero solution is stable. Thus, the zero solution is asymptotically stable and $D_{1}=\left\{\phi \in \mathrm{PC} \mid\|\phi\|_{\tau}<\alpha z_{2}\right\}$ is an asymptotically stable region of (2.2). The proof is complete. 
Similarly, we can obtain the following results for the case with $\alpha \geq 1$.

Theorem 3.5. Let $0<\theta \leq t_{k}-t_{k-1}$ for $k \in \mathbb{N}$. Assume that $\left(A_{1}\right)$ and $\left(A_{2}\right)$ with $\alpha \geq 1$ hold. If there exist two nonnegative constants $z_{1}<z_{2}$ such that for any $z \in\left(z_{1}, z_{2}\right)$

$$
\Psi_{2}(z):=\alpha p(z)+\left[\mu(f)+\frac{\ln \alpha}{\theta}\right] z<0,
$$

then $S_{2}=\left\{\phi \in \mathrm{PC} \mid\|\phi\|_{\tau} \leq z_{1}\right\}$ is an attracting set of (2.2) and $D_{2}=\left\{\phi \in \mathrm{PC} \mid\|\phi\|_{\tau}<\right.$ $\left.z_{2} / \alpha\right\}$ is an attracting basin of $S_{2}$.

Proof. Since $0<\theta \leq t_{k}-t_{k-1}$ and $\alpha \geq 1$, we replace the estimate (3.10) in the proof of Theorem 3.1 with

$$
W(t, s) \leq \alpha e^{[\mu(f)+\ln \alpha / \theta](t-s)}, \quad t \geq s \geq t_{0} .
$$

The rest of the proof is similar to one of the proof in Theorem 3.1 and we omit it here.

According to Theorem 3.5, we have the following.

Corollary 3.6. Let $t_{k}-t_{k-1} \geq \theta>0$ for $k \in \mathbb{N}$. Suppose that $\left(A_{1}\right)$ and $\left(A_{2}\right)$ with $\alpha \geq 1$ and $p(z)=\beta z+\gamma$ hold, where $\beta, \gamma \geq 0$. If $\alpha \beta+\ln \alpha / \theta+\mu(f)<0$, then $S_{1}=\left\{\phi \in \mathrm{PC} \mid\|\phi\|_{\tau} \leq\right.$ $\left.-\alpha \gamma[\alpha \beta+\ln \alpha / \theta+\mu(f)]^{-1}\right\}$ is a globally attracting set of (2.2).

Corollary 3.7. Let $x=0$ be a solution of (2.2). If all the conditions in Theorem 3.5 are satisfied except that the inequality (3.26) holds for $z \in\left(0, z_{2}\right)$, then the zero solution is asymptotically stable and $D_{2}=\left\{\phi \in \mathrm{PC} \mid\|\phi\|_{\tau}<z_{2} / \alpha\right\}$ is an asymptotically stable region.

\section{Illustrative examples}

Example 4.1. Consider a scalar nonlinear impulsive delay system:

$$
\begin{gathered}
\dot{x}(t)=a x(t)+b x^{2}(t-\tau)+c, \quad t \neq t_{k}, t \geq t_{0}=0, \\
\Delta x\left(t_{k}\right):=x\left(t_{k}\right)-x\left(t_{k}^{-}\right)=I_{k}\left(x\left(t_{k}^{-}\right)\right), \quad k \in \mathbb{N}
\end{gathered}
$$

where $b \neq 0,0<\theta \leq t_{k}-t_{k-1} \leq \varrho<+\infty,\left|x+I_{k}(x)\right| \leq \alpha|x|$. From $\left(\mathrm{A}_{1}\right)$ and $\left(\mathrm{A}_{2}\right), \mu(f)=a$, $p(z)=|b| z^{2}+|c|$. We discuss the attractiveness of (4.1) for the following cases.

Case $1(0<\alpha<1)$. Clearly, $\Psi_{1}(z)=(|b| / \alpha) z^{2}+(a+\ln \alpha / \varrho) z+|c| / \alpha$. If $a+\ln \alpha / \varrho<$ $-2 \sqrt{|b c|} / \alpha$, then the algebraic equation $\Psi_{1}(z)=0$ has two different nonnegative roots:

$$
z_{1,2}=\frac{-(a+\ln \alpha / \varrho) \mp \sqrt{(a+\ln \alpha / \varrho)^{2}-4|b c| / \alpha^{2}}}{2|b| / \alpha},
$$

and so $\Psi_{1}(z)<0$ for $z \in\left(z_{1}, z_{2}\right)$. According to Theorem 3.1 and Remark 3.2, $S_{1}=\{\phi \in$ $\left.\mathrm{PC} \mid\|\phi\|_{\tau} \leq z_{1}\right\}$ is an attracting set of (4.1) and $D_{1}=\left\{\phi \in \mathrm{PC} \mid\|\phi\|_{\tau}<\alpha z_{2}\right\}$ is an attracting basin of $S_{1}$. Especially, when $c=0$ and $a+\ln \alpha / \varrho<0$, it follows from Corollary 3.4 that the zero solution of (4.1) is asymptotically stable and an asymptotically stable region $D_{1}=\left\{\phi \in \mathrm{PC} \mid\|\phi\|_{\tau}<-\left(\alpha^{2} /|b|\right)(a+\ln \alpha / \varrho)\right\}$. 


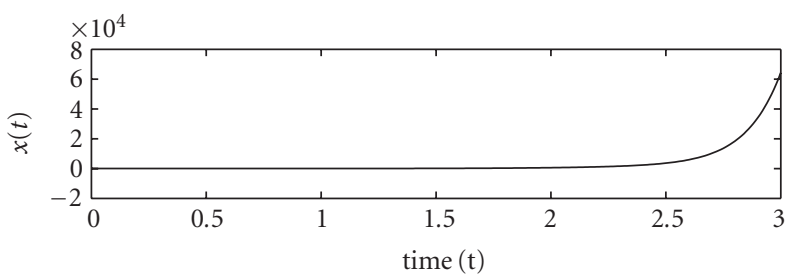

(a)

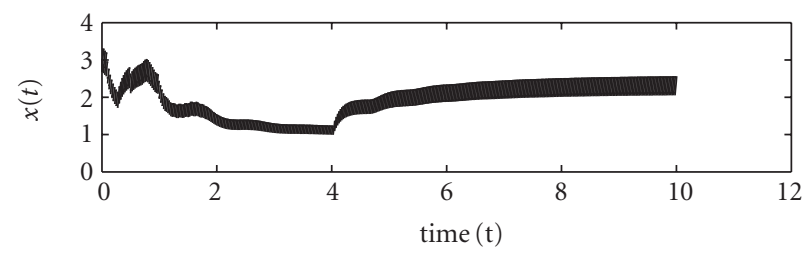

(b)

Figure 4.1. The trajectory of (4.1): (a) no impulses; (b) impulsive effects.

Case $2(\alpha \geq 1)$. Clearly, $\Psi_{2}(z)=\alpha|b| z^{2}+(a+\ln \alpha / \theta) z+\alpha|c|$. If $a+\ln \alpha / \theta<-2 \alpha \sqrt{|b c|}$, then the algebraic equation $\Psi_{2}(z)=0$ has two different nonnegative roots:

$$
z_{1,2}=\frac{-(a+\ln \alpha / \theta) \mp \sqrt{(a+\ln \alpha / \theta)^{2}-4 \alpha^{2}|b c|}}{2 \alpha|b|},
$$

and so $\Psi_{2}(z)<0$ for $z \in\left(z_{1}, z_{2}\right)$. According to Theorem 3.5, $S_{2}=\left\{\phi \in \mathrm{PC} \mid\|\phi\|_{\tau} \leq z_{1}\right\}$ is an attracting set of (4.1) and $D_{2}=\left\{\phi \in \mathrm{PC} \mid\|\phi\|_{\tau}<z_{2} / \alpha\right\}$ is an attracting basin of $S_{2}$. Especially, when $c=0$ and $a+\ln \alpha / \theta<0$, it follows from Corollary 3.7 that the zero solution of (4.1) is asymptotically stable and an asymptotically stable region $D_{2}=\{\phi \in$ PC $\left.\mid\|\phi\|_{\tau}<-\left(1 / \alpha^{2}|b|\right)(a+\ln \alpha / \theta)\right\}$.

Take $a=b=1, c=10, \tau=1, I_{k}(x)=-0.2 x$, and $t_{k}=t_{k-1}+0.025$. From Case 1 with $\alpha=0.8$ and $\varrho=0.025$, we have $z_{1}=2.9449, z_{2}=3.3956$, and so $S_{1}=\left\{\phi \in \mathrm{PC} \mid\|\phi\|_{\tau} \leq\right.$ $2.9449\}$ is an attracting set of the impulsive system (4.1), $D_{1}=\left\{\phi \in \mathrm{PC} \mid\|\phi\|_{\tau}<\alpha z_{2}=\right.$ $2.7165\}$ is an attracting basin of $S_{1}$. However, any solution of the corresponding continuous delay system (i.e., $\Delta x\left(t_{k}\right)=0$ in (4.1)) is unbounded. Figure 4.1 shows the different asymptotical behaviors between the impulsive system and the corresponding continuous system under the initial condition $x(t)=2.7, t \in[-1,0]$.

Example 4.2. Consider a two dimensional impulsive delay system

$$
\begin{gathered}
\dot{x}_{1}(t)=x_{1} \sin x_{1}(t)+x_{2}(t-1) \cos \left(x_{1}(t-1)\right)+0.5 \sin e^{t}, \quad t \geq t_{0}=0, \\
\dot{x}_{2}(t)=x_{2} \cos x_{2}(t)-x_{1}(t-1) \sin \left(x_{2}(t-1)\right)+0.5 \cos e^{t}, \quad t \neq t_{k}, \\
\Delta x_{1}:=x_{1}\left(t_{k}^{+}\right)-x_{1}\left(t_{k}^{-}\right)=-0.4 x_{1}\left(t_{k}^{-}\right), \quad t_{k}=t_{k-1}+0.15, \\
\Delta x_{2}:=x_{2}\left(t_{k}^{+}\right)-x_{2}\left(t_{k}^{-}\right)=-0.4 x_{2}\left(t_{k}^{-}\right), \quad k \in \mathbb{N} .
\end{gathered}
$$




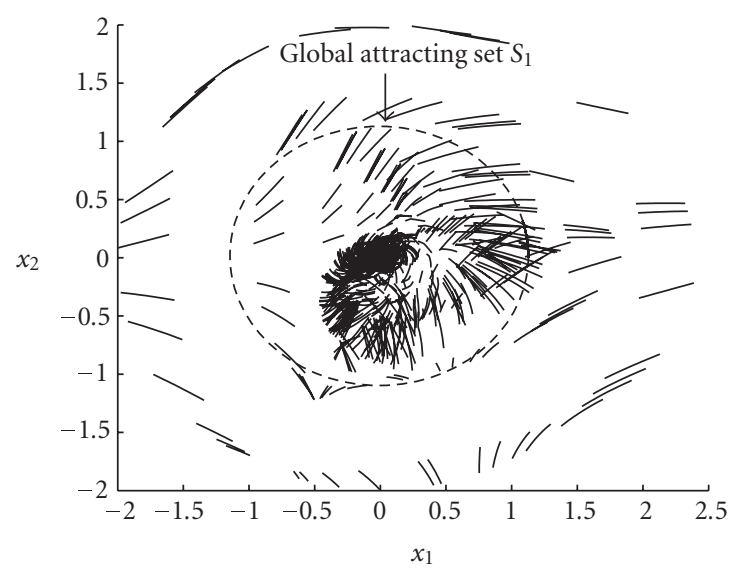

Figure 4.2. The global attracting set of the impulsive delay system (4.4).

Letting $f(x)=\left(x_{1} \sin x_{1}, x_{2} \cos x_{2}\right)^{T}, g(t, x)=\left(x_{2} \cos x_{1}+0.5 \sin e^{t},-x_{1} \sin x_{2}+0.5 \cos e^{t}\right)^{T}$, then $\|f(x)\| \leq\|x\|,\|g(t, x)\| \leq\|x\|+0.5$, where $\|\cdot\|$ is the 2-norm of the vector. From Lemma 2.4, we can calculate the condition parameters in Theorem 3.1:

$$
\begin{gathered}
\mu(f) \leq 1, \quad p(z)=z+0.5, \quad \alpha=0.6, \quad \varrho=0.15 \\
\Psi_{1}(z) \leq-0.7388 z+0.8333<0, \quad z \in(1.1279,+\infty) .
\end{gathered}
$$

It follows from Theorem 3.1 (or Corollary 3.3) that $S_{1}=\left\{\phi \mid\|\phi\|_{\tau} \leq 1.1279\right\}$ is a global attracting set of (4.4). Figure 4.2 shows the attractivity of the impulsive delay system (4.4) under the different initial conditions.

\section{Acknowledgment}

The work is supported by the National Natural Science Foundation of China under Grant no. 10371083.

\section{References}

[1] D. D. Bănov and P. S. Simeonov, Systems with Impulse Effect. Stability, Theory and Applications, Ellis Horwood Series: Mathematics and Its Applications, Ellis Horwood, Chichester, 1989.

[2] D. D. Bănov and I. M. Stamova, Existence, uniqueness and continuability of solutions of impulsive differential-difference equations, Journal of Applied Mathematics and Stochastic Analysis 12 (1999), no. 3, 293-300.

[3] S. D. Borisenko, V. I. Kosolapov, and A. Yu. Obolenski1̌, Stability of Processes under Continuous and Discrete Perturbations, "Naukova Dumka", Kiev, 1988.

[4] V. B. Kolmanovskii and A. Myshkis, Introduction to the Theory and Applications of FunctionalDifferential Equations, Mathematics and Its Applications, vol. 463, Kluwer Academic, Dordrecht, 1999.

[5] V. B. Kolmanovskii and V. R. Nosov, Stability of Functional-Differential Equations, Mathematics in Science and Engineering, vol. 180, Academic Press, London, 1986. 
[6] V. Lakshmikantham, D. D. Baĭnov, and P. S. Simeonov, Theory of Impulsive Differential Equations, Series in Modern Applied Mathematics, vol. 6, World Scientific, New Jersey, 1989.

[7] V. Lakshmikantham and S. Leela, Differential and Integral Inequalities: Theory and Applications. Vol. I: Ordinary Differential Equations, Academic Press, New York, 1969.

[8] _ Differential and Integral Inequalities: Theory and Applications. Vol. II: Functional, Partial, Abstract, and Complex Differential Equations, Mathematics in Science and Engineering, vol. 55-II, Academic Press, New York, 1969.

[9] S. Li, D. Xu, and H. Zhao, Stability region of nonlinear integrodifferential equations, Applied Mathematics Letters 13 (2000), no. 2, 77-82.

[10] X. Liu and G. Ballinger, Uniform asymptotic stability of impulsive delay differential equations, Computers \& Mathematics with Applications 41 (2001), no. 7-8, 903-915.

[11] _ Existence and continuability of solutions for differential equations with delays and statedependent impulses, Nonlinear Analysis. Series A: Theory and Methods 51 (2002), no. 4, 633647.

[12] X. Liu, K. L. Teo, and Y. Zhang, Absolute stability of impulsive control systems with time delay, Nonlinear Analysis 62 (2005), no. 3, 429-453.

[13] A. N. Michel, J. A. Farrell, and W. Porod, Qualitative analysis of neural networks, IEEE Transactions on Circuits and Systems 36 (1989), no. 2, 229-243.

[14] H. Qiao, J. Peng, and Z.-B. Xu, Nonlinear measures: a new approach to exponential stability analysis for Hopfield-type neural networks, IEEE Transactions on Neural Networks 12 (2001), no. 2, 360-370.

[15] A. V. Razgulin, The attractor of the delayed functional-differential diffusion equation. Numerical methods in mathematical physics, Computational Mathematics and Modeling 8 (1997), no. 2, $181-186$.

[16] A. M. Samoillenko and N. A. Perestyuk, Impulsive Differential Equations, World Scientific Series on Nonlinear Science. Series A: Monographs and Treatises, vol. 14, World Scientific, New Jersey, 1995.

[17] D. D. Šiljak, Large-Scale Dynamic Systems. Stability and Structure, North-Holland Series in System Science and Engineering, vol. 3, North-Holland, New York, 1979.

[18] D. Xu and H. Zhao, Invariant set and attractivity of nonlinear differential equations with delays, Applied Mathematics Letters 15 (2002), no. 3, 321-325.

[19] J. Yan and J. Shen, Impulsive stabilization of functional-differential equations by LyapunovRazumikhin functions, Nonlinear Analysis. Series A: Theory and Methods 37 (1999), no. 2, 245255.

[20] J. S. Yu, Stability for nonlinear delay differential equations of unstable type under impulsive perturbations, Applied Mathematics Letters 14 (2001), no. 7, 849-857.

[21] Y. Zhang and J. Sun, Eventual practical stability of impulsive differential equations with time delay in terms of two measurements, Journal of Computational and Applied Mathematics 176 (2005), no. $1,223-229$.

Zhichun Yang: Mathematical College, Sichuan University, Chengdu 610064, China

Current address: Department of Mathematics, Chongqing Normal University,

Chongqing 400047, China

E-mail address: zhichy@yahoo.com.cn

Daoyi Xu: Mathematical College, Sichuan University, Chengdu 610064, China

E-mail address: daoyixucn@yahoo.com 


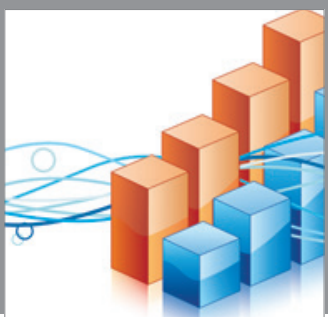

Advances in

Operations Research

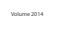

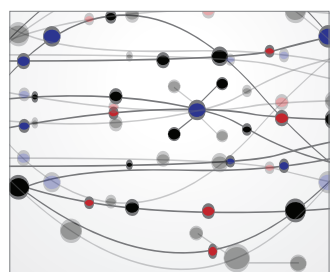

\section{The Scientific} World Journal
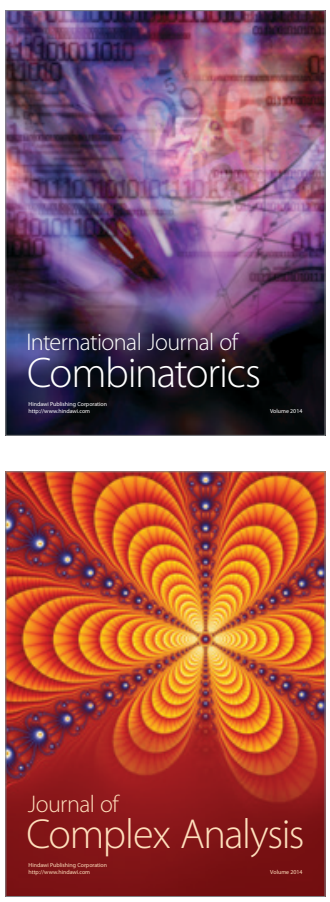

International Journal of

Mathematics and

Mathematical

Sciences
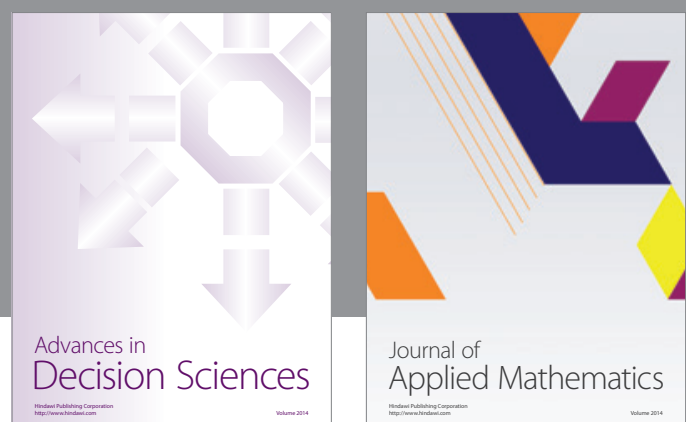

Journal of

Applied Mathematics
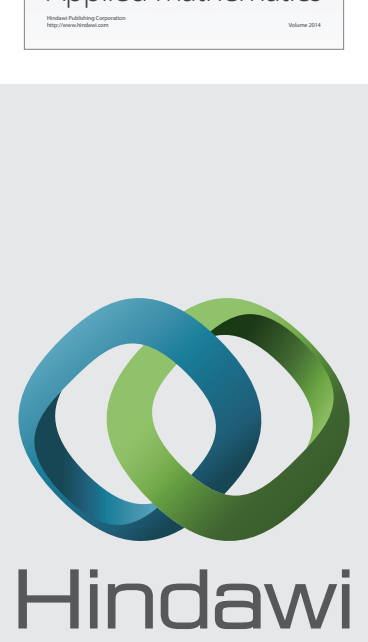

Submit your manuscripts at http://www.hindawi.com
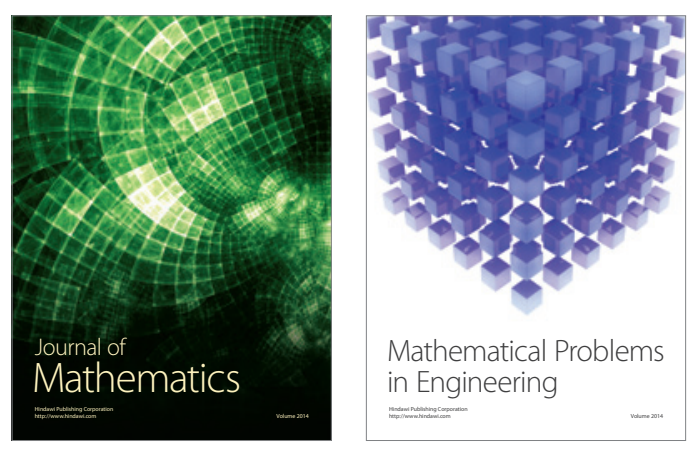

Mathematical Problems in Engineering
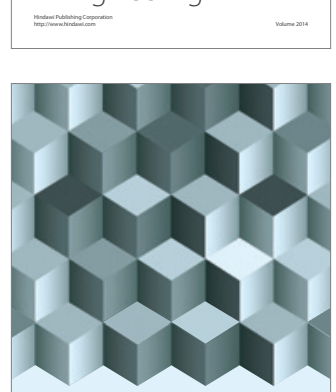

Journal of

Function Spaces
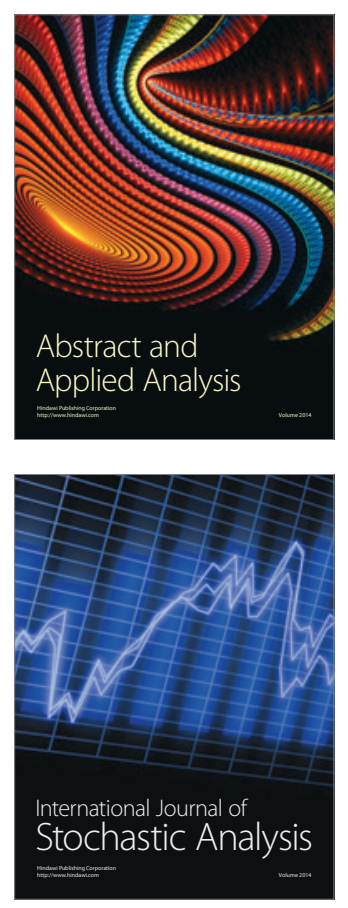

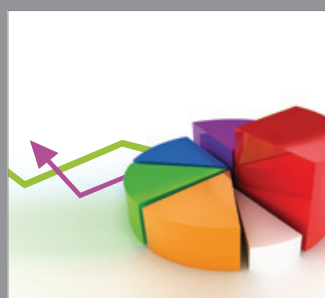

ournal of

Probability and Statistics

Promensencen
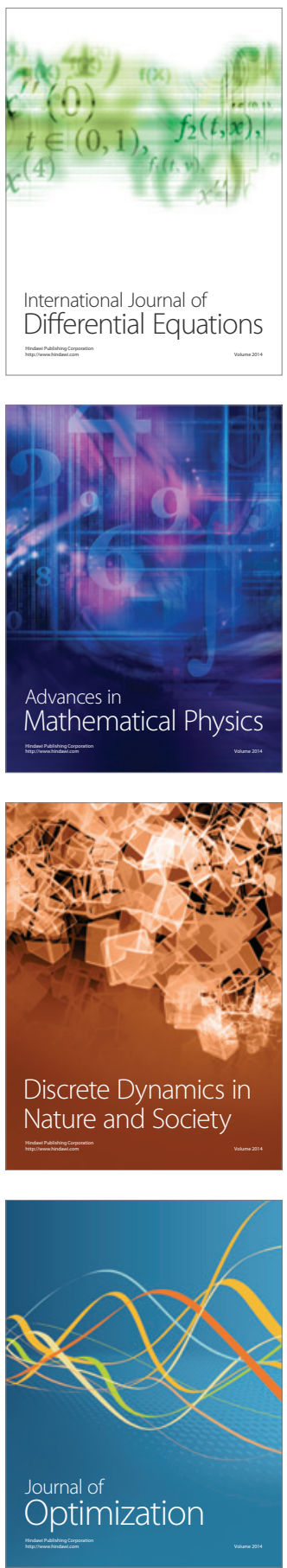